3. Pigeon C, llyin G, Courselaud B, et al. A new mouse liver-specific gene, encoding a protein homologous to human antimicrobial peptide hepcidin, is overexpressed during iron overload. J Biol Chem 2001; 276 : 7811-9.

4. Roetto A, Papanikolaou G, Politou M, et al. Mutant antimicrobial peptide hepcidin is associated with severe juvenile hemochromatosis. Nat Genet 2002; 33: 21-2.
5. Nicolas G, Benounn M, Porteu A, et al. Severe iron deficiency anemia in transgenic mice expressing liver hepcidin. Proc Natl Acad Sci USA 2002; 99 : 4596-601.

6. Nemeth $\varepsilon$, Tuttle MS, Powelson J, et al. Hepcidin regulates cellular iron efflux by binding to ferroportin and inducing its internalization. Science Express 28 octobre 2004.
7. Nicolas G, Chauvet C, Viatte L, et al. The gene encoding the iron regulatory peptide hepcidin is regulated by anemia, hypoxia, and inflammation. J Clin Invest 2002; 110: 1037-44.

8. Nemeth $\varepsilon$, Valore $\varepsilon V$, Territo $M$, et al. Hepcidin, a putative mediator of anemia of inflammation, is a type II acute-phase protein. Blood 2003; 101 : 2461-3.

\title{
NOUVELLE
}

\section{Recyclage polarisé et formation de la synapse immune dans les lymphocytes T}

Andrés Alcover, Maria Isabel Thoulouze, Thierry Galli
> Parmi les interfaces de communication entre les cellules du système immunitaire, la synapse immune est l'une des mieux étudiées. Induite par la reconnaissance de l'antigène, celle-ci se forme à la jonction entre le lymphocyte $T$ et la cellule présentatrice d'antigène. La synapse immune se caractérise par l'accumulation, puis la réorganisation à l'interface cellulaire, de divers récepteurs de surface - dont le récepteur de l'antigène des lymphocytes $T$ (récepteur T) -, de protéines du cytosquelette et de protéines de signalisation cellulaire. Sa description dans les années 1990 a soulevé des questions importantes, telles que l'universalité de son organisation, la nature des mécanismes qui mènent à sa formation et le rôle de cette structure très organisée dans le processus d'activation des lymphocytes $T$ [1]. Une question centrale s'est alors posée: comment les récepteurs membranaires sont-ils transportés et s'accumulent-ils dans la synapse immune? Plusieurs mécanismes interviendraient: les récepteurs se déplaceraient latéralement dans la membrane, soit par diffusion passive, soit par un mouvement actif facilité par le cytosquelette d'actine et par des moteurs moléculaires de type myosine [2] (Figure 1A). Les travaux récents de V. Das et al. [3] ont permis d'identifier un nouveau mécanisme de transport du récepteur T vers la synapse immune (Figure 1B). Celuici met en jeu deux phénomènes différents: la propriété du récepteur $\mathrm{T}$ d'être internalisé et recyclé vers la membrane plasmique, et la capacité des lymphocytes $T$ de polariser leur compartiment vésiculaire d'endocytose précoce et de recyclage vers la synapse immune. Des récepteurs $T$ qui transitent par des endosomes peuvent ainsi être acheminés vers la synapse immune où ils sont recyclés vers la membrane plasmique. Ces travaux démontrent notamment que ce recyclage polarisé de récepteurs conduit à leur accumulation dans la synapse immune. Pour mettre en évidence ce mécanisme, des techniques de microscopie en temps réel ont d'abord été utilisées. Les résultats indiquent que des vésicules d'endocytose contenant des récepteurs $T$ sont transportées vers la zone de contact entre le lymphocyte $T$ et la cellule présentatrice d'antigène, et qu'elles recyclent les récepteurs dans cette zone de la cellule. Ces expériences démontrent en outre que ce type de transport est nécessaire pour que le récepteur $T$ s'accumule au niveau de la synapse immune. L'exocytose des vésicules contenant les récepteurs $T$ est sous le contrôle des protéines fusogènes SNARE, cellubrévine, syntaxine 4 et SNAP23, homologues des protéines SNARE des synapses neuronales. Ainsi, la quantité de récepteur $T$ accumulé dépend à la fois de la capacité du récepteur d'être internalisé, du transport de vésicules le long des microtubules, et du recyclage et de la fusion de vésicules d'exocytose avec la membrane plasmique. Les lymphocytes $T$ peuvent ainsi transporter des récepteurs d'un endroit à un autre de la membrane plasmique par une voie intracellulaire impliquant des endosomes [3].

La mise en jeu d'un tel mécanisme s'étend à bien d'autres transports que celui des récepteurs T. II ferait partie d'un mécanisme plus général qui se met en place dans les lymphocytes T consécutivement à la reconnaissance antigénique. Par exemple, le récepteur de la transferrine, qui est recyclé continuellement pour transporter du fer du milieu extracellulaire vers l'intérieur de la cellule, suit le même parcours et s'accumule également dans la synapse immune. Hormis les récepteurs membranaires, ce mécanisme pourrait également impliquer des protéines de signalisation associées à la membrane plasmique, comme la tyrosine kinase Lck ou l'adaptateur LAT (linker of activation of $T$ cell). Ces protéines transitent également par un compartiment vésiculaire qui contient des protéines endosomiques, compartiment qui se polarise et semble fusionner avec la membrane au niveau de la synapse immune $[4,5]$. 
Le transport polarisé de protéines utilisant le compartiment endosomique n'est pas spécifique des lymphocytes T. II constitue un mécanisme conservé, utilisé lors de divers processus cellulaires. Lors de la migration cellulaire ou pendant la phagocytose, par exemple, les endosomes de recyclage se dirigent respectivement vers le front de migration ou vers la coupe phagocytaire, où ils apportent des composants membranaires $[6,7]$. Dans les neurones, des endosomes de recyclage peuvent également acheminer des récepteurs postsynaptiques vers la membrane plasmique de la synapse et participer ainsi au renforcement de la transmission synaptique caractéristique de la potentialisation à long terme [8]. La mise en évidence de ce mode de transport lors de la formation de la synapse immune soulève à son tour de nombreuses questions: quelles sont les protéines régulatrices qui « connectent »le signal transmis par le récepteur T à la suite de la reconnaissance antigénique ? À quel stade contrôlent-elles ce transport? Au niveau de la polarisation du cytosquelette, du transport de vésicules ou bien encore de leur fusion au niveau de la synapse immune? Enfin, ces protéines régulatrices interviennent-elles aussi dans le contrôle d'autres types de trafic polarisé - celui des granules cytotoxiques [9], des vésicules de sécrétion issues de l'appareil de Golgi [10] ou bien encore celui des endosomes tardifs ? La mise en évidence d'un rôle important de l'exocytose et des protéines SNARE rapproche encore un peu plus la synapse immunologique de la synapse neuronale, site d'un trafic membranaire des plus intenses. $\diamond$ Activation-induced polarized recycling targets T cell receptors to the immunological synapse

\section{RéFÉRENCES}

1. Huppa JB, Davis MM. T-cell-antigen recognition and the immunological synapse. Nat Rev Immunol 2003; $3: 973-83$.

2. Wülfing C, Davis MM. A receptor/cytoskeletal movement triggered by costimulation during T cell activation. Science $1998 ; 282$ : 2266-9.
3. Das V, Nal B, Dujeancourt A, et al.

Activation-induced polarized recycling synapse. Involvement of SNARE complexes. Immunity $2004 ; 20$ : 577-88.

4. Ehrlich LIR, Ebert PJR, MF Krummel, et al. Dynamics pf p56lck translocation to the T cell immunological synapse following agonist and antagonist stimulation. Immunity $2002 ; 17: 809-22$.

5. Bonello G, Blanchard N, Montoya MC, et al. Dynamic recruitment of the adaptor protein LAT: LAT exists in recruitment. J Cell Sci 2003 ; 117 : 1009-16. targets $T$ cell receptors to the immunological two distinct intracellular pools and controls its own

6. Bretscher MS, Aguado-Velasco C. Membrane traffic during cell locomotion. Curr Opin Cell Biol 1998 ; $10: 537-41$.

7. Booth S, Trimble WS, Grinstein S. Membrane dynamics in phagocytosis. Semin Immunol $2001 ; 13$ : 357-64.

8. Park M, Penick EC, Edwards JG, et al. Recycling endosomes supply AMPA receptors for LTP. Science $2004 ; 305: 1972-5$.

9. Clark R, Griffiths GM. Lytic granules, secretory Iysosomes and disease. Curr Opin Immunol 2003 ; $15: 516-21$.

10. Kupfer A, Mosmann TR, Kupfer H. Polarized expression of cytokines in cell conjugates of helper T cells and splenic B cells. Proc Natl Acad Sci USA 1991; 88: 775-9.

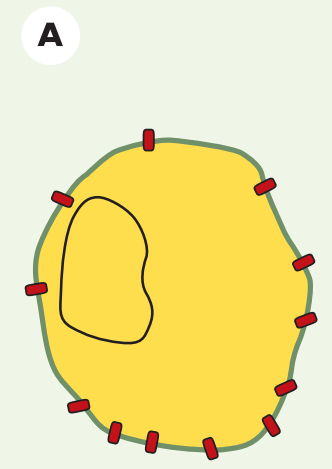

Cellule T non activée

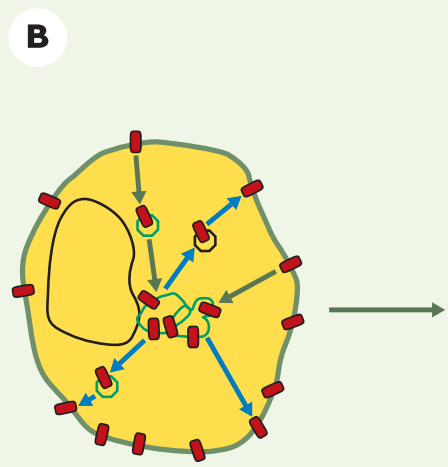

Cellule T non activée

Ligand du TCR

$\mathrm{CMH}$-antigène

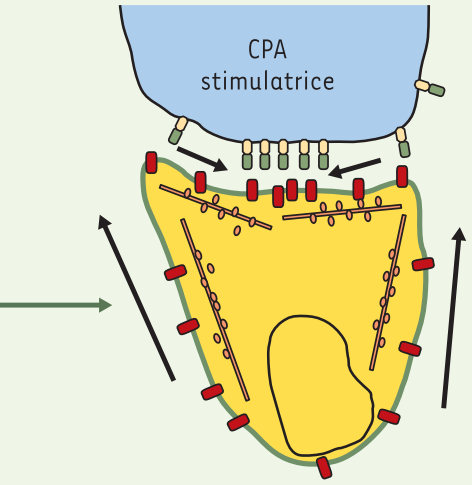

Cellule T activée

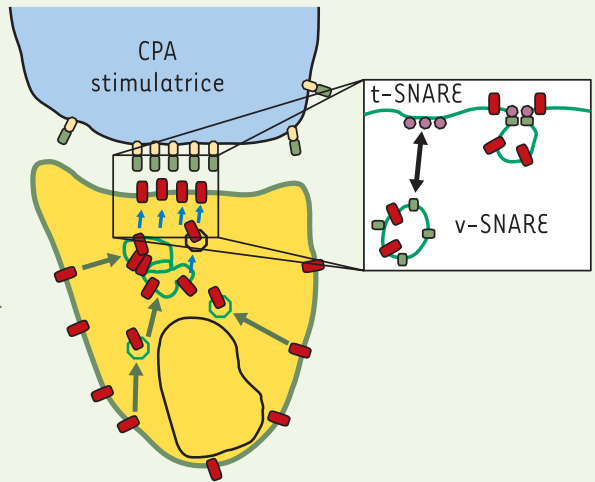

Cellule T activée

o t-SNARE
○-SNARE
Figure 1. Modèle de ciblage du récepteur T (TCR) vers la synapse immune par recyclage polarisé. $A$. Les molécules de surface peuvent être dirigées vers la zone de contact avec la cellule présentatrice d'antigène (CPA) directement à partir de la surface cellulaire, soit par diffusion passive, soit par un mécanisme actif mettant en jeu le cytosquelette d'actine et les moteurs de type myosine. B. Les molécules de TCR, ainsi que les autres récepteurs qui sont recyclés tels que le récepteur de la transferrine, peuvent être adressés à la synapse immune via des endosomes de recyclage. Dans les cellules T non activées (à gauche), l'internalisation (flèches vertes) et le recyclage (flèches bleues) s'opèrent de manière aléatoire d'une région à l'autre de la membrane plasmique. En revanche, après activation par une CPA, l'internalisation peut toujours s'opérer de manière aléatoire (flèches vertes), mais l'exocytose des vésicules de recyclage (flèches bleues) prend place préférentiellement au niveau de la zone de contact avec la CPA. Le regroupement des t-SNARE, puis l'arrimage et la fusion des vésicules de recyclage contenant la v-SNARE cellubrévine semblent se produire à ce niveau (encart): SNAP: soluble NSF-attachment protein; NSF: N-ethylmaleimide sensitive fusion protein; t-SNARE : target-membrane SNAP receptor; $v$-SNARE: vesicle-membrane SNAP receptor. 